\title{
Symmetry States of the Physical Space: An Expanded Reference Frame for Understanding Human Consciousness
}

\author{
Nisha J. Manek, MD
}

\begin{abstract}
"A radical mutation in the quality of human consciousness will take place. We are concerned with preparing the ground for the emergence of a new human race."

-Vimala Thakar

"Man is undergoing evolution. First, he was Homo erectus. Then, he became Homo sapiens. Now, he is evolving into Homo spiritus."
\end{abstract}

—David R. Hawkins, MD, PhD

\begin{abstract}
A remarkable phenomenon is taking place around the globe, one that I have been fortunate enough to witness and in which to participate. The relics of the historical Buddha, also known as Siddhartha or Shakyamuni Buddha, still survive today over 2500 years since his enlightenment, and, for the first time in history, are traveling throughout the world. In common Buddhist practice, relics are highly venerated and treasured remains of realized Masters. It is very rare for relics to travel from city to city and be available for viewing by the general public. The Buddha relic tour is demonstrating that a direct experience of the spiritual state is not mysterious, nor is it for a select few. The spiritual state, here defined as a universal theme of unconditional love, is a component of human evolutionary unfoldment, a process through which thousands of human beings have passed, and through which thousands more will pass. We are "waking up" as a species. Consequently, more information is required about this transformation of human consciousness. The Buddha relics offer us a priceless means by which we can obtain a richer perspective about the nature of human consciousness, spiritual realities such as love, and ultimately understanding ourselves.
\end{abstract}

\section{Introduction}

$\mathbf{H}$ UMAN BEINGS INTUITIVELY TURN to spirituality for healing. According to Barnes et al., prayer for self and prayer for others are the most often utilized alternative therapies by adults in the United States. ${ }^{1}$ In parallel, there has been a tremendous increase in scientific interest regarding the relationship between spirituality and health. ${ }^{2}$ Spirituality, here defined as unconditional love, and its correlates such as compassion and altruism, are receiving serious attention in medical research. From research of the "relaxation response," ${ }^{3,4}$ mindfulness meditation, ${ }^{5}$ and psychoneuroimmunology 6,7 emerges a neurologically based rationale for cognitive techniques for the development of self-awareness. The mechanisms by which the emotional and cognitive changes of meditation can be of therapeutic value have been explored, and the synergistic advantages of the combination of psychotherapy and meditation have been extensively discussed in the medical literature. ${ }^{8,9}$ Furthermore, utilizing sophisticated functional magnetic resonance imaging of the brain, one can see changes in the central nervous system to a stunning level of accuracy in adept people such as a high Lama. ${ }^{10}$ We also know that regular meditation can positively modulate the endocrine and immune systems and forms an important basis for mind-body medicine. ${ }^{11,12}$ Human consciousness effects in the form of intention have also been the subject of much research; for example, the mood-elevating effects of chocolate can be enhanced with intention! $!^{13}$ These and many other studies have enriched our understanding of the inherent healing and resilient capacities of our bodies and minds. The mechanisms of meditation that medical science has so carefully demonstrated seemed clear to me: Practice meditation, mindfulness, compassion, and qualities of love and, over time, our minds, emotions, and body will be

Division of Rheumatology, Department of Medicine, Mayo Clinic, Rochester, MN. 
healthier. This seemed reasonable enough until one astonishing afternoon I visited the Buddha relics, which made me dig deeper into the science of human intention. The search for the meaning of my experience was not an easy task, because intention or love or any such emotion is radically subjective. Yet, as a physician and scientist, I hold objectivity paramount. I recognize that subjectivity is a source of great difficulty for the scientific community and may be a problem for many scientists and readers of this article. The fact is, however, that we cannot escape subjectivity. Even as I formulate an objective opinion about any personal or scientific information, it still is based on my subjective viewpoint.

My inquiry has led me to examine the relics, delve into consciousness research, and experience an unfolding adventure in physics. I believe that the Buddha relics offer us a priceless means by which we can obtain a richer perspective about the nature of the spiritual state, and ultimately that of the universal theme of unconditional love; in this examination I have tried to avoid the polarities of intellectual skepticism and enthusiastic credulity. In exploring the nature of consciousness and the spiritual domain, and bridging to what we know today in science, I have stepped out of my comfort zone and crossed the lines of my academic expertise. I offer my insights to open-minded fellow scientists who may wish to investigate these matters for themselves. I will discuss what the relics might mean for medicine, our patients, and ultimately, for understanding ourselves.

\section{The Buddha Relic Tour}

A relic is the remains, or a personal item, of great spiritual significance, carefully preserved and treasured as a tangible memorial of a realized Master or spiritual teacher. In the Tibetan Buddhist tradition, a Master may choose to leave behind crystal or pearl-like objects during the cremation of the body. The relics of the historical Buddha, also known as Shakyamuni Buddha, were safeguarded and taken out of Tibet during China's invasion. A new home for the sacred relics is now under construction in Kushinagar, northern India, in the form of a 500-foot statue of the Maitreya Buddha ("loving-kindness Buddha"). ${ }^{14}$ In the meantime, it was the vision of Lama Zopa Rinpoche, spiritual director of the Maitreya Project, that the relics travel throughout the world so that people everywhere could benefit from the radiant blessings the relics emanate. The tour includes relics of Shakyamuni Buddha (donated by His Holiness the 14th Dalai Lama), the Buddha's closest disciples, and well-known Buddhist Masters from different traditions. Since the tour began in 2001, the relics have visited churches, temples, educational institutions, as well as hospitals in over 50 countries. More than 2 million people around the world have viewed the relics. The tour is nonsectarian and free to all visitors.

\section{Personal Experiences of the Buddha Relics}

Through the gift of circumstance, on a quiet Sunday afternoon in September 2009, my brother and I visited the Heart Shrine Buddha relic tour at the Gyuto monastery in Minneapolis. I am not of the Buddhist faith and as such I went with a curious, or what one may call a "tourist" mindset. I never imagined what a profound effect my visit would have on me. In retrospect, it was an astonishing and life-changing experience.

Upon entering the monastery, I immediately felt an intense state of awareness, or Presence. It was as though the Buddha himself was present. The state was nonverbal, vast, and profound; it was exquisitely gentle and yet like a rock. There was a silence and a state of peace that seemed limitless. For me, the essence of time ceased to exist. My mind became quieter: for in place of the usual internal conversation was wonder; and curiosity had given way to amazement. I felt a tangible radiation of exquisite energy flowing from the relics to my heart center. It was highly private and personal, and yet conveyed an immense sense of Oneness or unity with everyone and everything. It had no counterpart in ordinary experience.

For several days afterward, I had a sense of not "being home," that my home was the state that I had experienced at the relic tour. I had to energize back to dealing with the business at hand. To sequentially analyze data or information was arduous. Over some 4 to 5 days, this feeling gradually passed. However, on a deeper and more subtle level, I was not the same any longer. Belief in God, as I discovered, and the experience with God are profoundly different. The subjective experience was discussed with a few of my patients who sensed that their doctor was "different"; they were at once fascinated and confirmed my descriptions in that it was familiar from their own spiritual studies of the bible or church practices. Other than that, I did not share my experience with anyone as there was no context available from which I could meaningfully describe it.

\section{The Heart Shrine Buddha Relic Tour Comes to Southern California}

The immensity of my inner experience was such that my brother and I made a firm intention to host the relic tour at our family home in Southern California. Through the grace and kindness of the Maitreya Project, my family hosted the Buddha relic tour over 3 days in the summer of 2010. I was once again given an opportunity to experience and confirm that which I had encountered 9 months previously in Minnesota. As hundreds of people came through our home, and from the messages and e-mails we subsequently received, it became clear that a shift of perception was occurring for the visitors. It was very personal, very powerful, very meaningful; it did not matter what spiritual tradition they came from, if they regularly meditated or not, or if they even believed in a higher being. Many visitors reported a feeling of deep peace, and many others were overcome with tears. For some, physical ailments resolved. It did not seem important how serious or chronic the disease was, but the body healed. In following one of these cases with the treating physician, he shared that in his medical opinion, the resolution of the illness, which involved recovery of renal impairment and ceasing dialysis in the diabetic patient, was unexpected. * As with any medical healing, the natural course of disease may also account for the improvement. The patient remains well, 11 months after the relic tour. Many unusual phenomena have been recorded in relation to relic tours (Fig. 1).

\footnotetext{
*Franklin Cabebe, MD, Glendora, CA [personal communication, August 2010 and May 2011].
} 


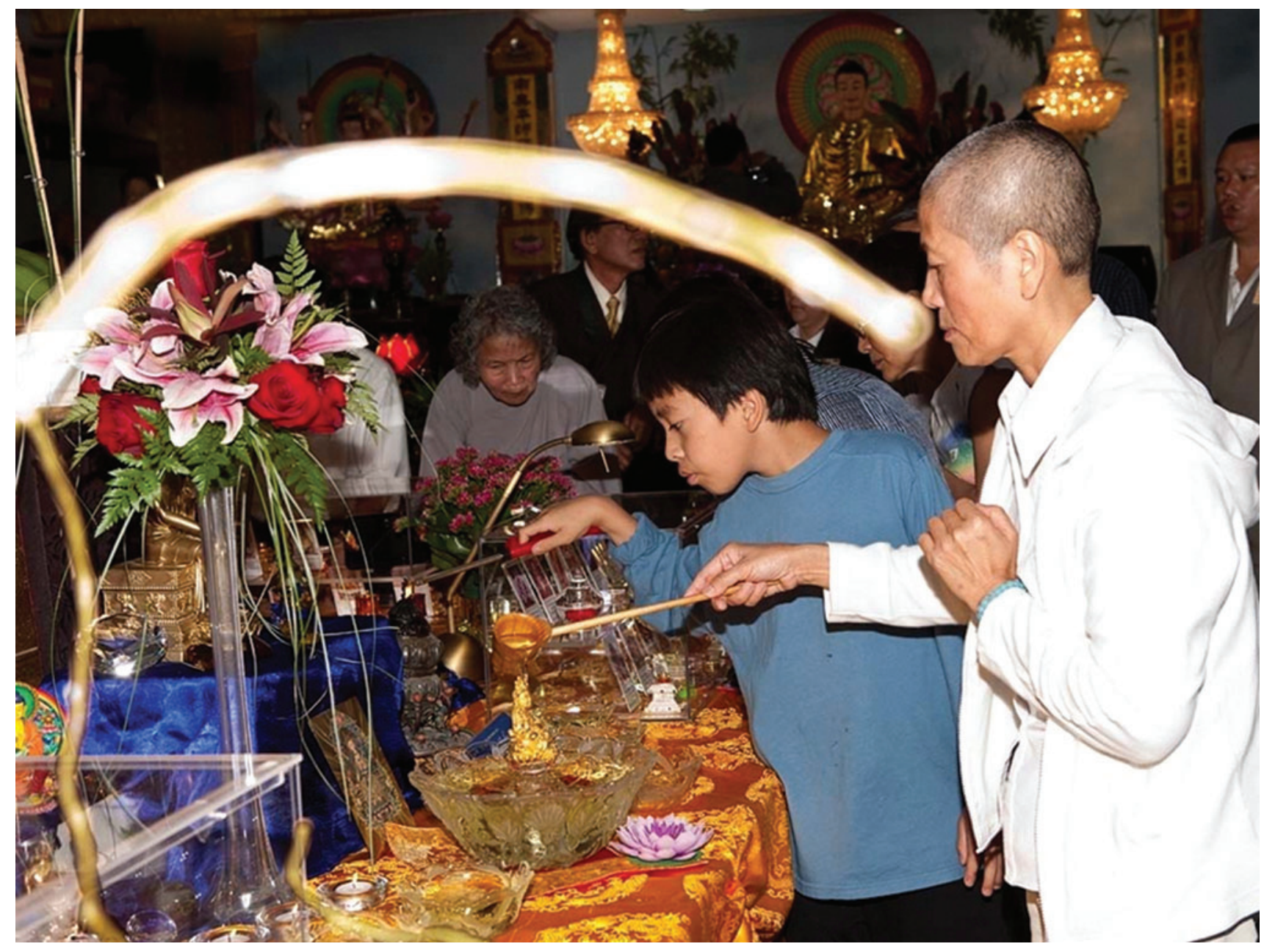

FIG. 1. Picture of the Buddha relic tour taken in Santa Ana, California, July 2010. The picture was taken by a professional photographer who was commissioned to record the relic tour at a Vietnamese temple in Santa Ana, CA, 1 week before the relics came to the Manek home. The picture was taken with a standard Canon professional camera at approximately 12:00 noon on July 25, 2010. The lights that are so clearly visible in the picture were not visible to the naked eye in the space of the temple. One can see a golden translucent light to the lower left of the picture, which then emerges into a strong band of luminescent orbs arching over the altar table. How is this image possible and how does it inform us of the nature of the space in the temple during the relic tour? All these reports from many different sources and independent personal perspectives convinced me that the Buddha relics offer a unique opportunity to understanding a different and powerful reality, one in which shifts can potentially occur in the emotional, mental, and physical domains of reality. (Photograph with permission and courtesy of Ms. Julayne Luu, Julayne Luu Photography, Midway City, CA; web address: www.klphotostudio.com).

\section{What is the Nature of the Buddha Relics?}

The core question is: what is the nature of the actual energies that so tangibly radiate from the relics? Is the energy a new force in traditional science? I do not believe the energy the relics emanate represents a new or unknown force as I know of no scientific force such as gravity or electromagnetic radiation that can induce such strong emotional and transformational qualities in people in so a short a time. The energy is, in my view, loving kindness. Then, how is it possible for formless qualities of consciousness, such as loving kindness, to be encapsulated in a form, such as a relic? Can effects of human consciousness be quantifiable in some way? In my explorations of other branches of science, I feel physics may give some important clues as to the nature of this higher reality.

\section{Expanding Our Paradigm for Understanding Nature}

Newtonian or classical physics with a distance-time reference frame has been the predominant academic scientific worldview for over 400 hundred years. It is Newtonian physics that enabled humankind to put a man on the moon, and in our everyday macroworld reality we constantly deal with this paradigm. ${ }^{15}$ However, human qualities such as consciousness, intention, and emotion are not incorporated into this standard model. It was not until Nobel Laureates Werner Heisenberg and Erwin Schrödinger founded the now well-accepted Quantum Theory in the early part of the 20th century that science acknowledged the effect of human action. Heisenberg and Schrödinger called this the "observer effect."16 In science, the observer effect means that the act of observing will influence the phenomenon 
being observed. ${ }^{16}$ However, it is not as simple as that and we must dig deeper. Schrödinger's famous wave equation describes how the quantum state of a system-interpreted as the probability of a particle being detected at a certain locationevolves over time. ${ }^{17}$ The mathematics of Schrödinger's wave equation is a second-order differential equation and is thought to require energy in space and time for the collapse of the wave function. ${ }^{17}$

If we pause a moment here, we see some problems with Quantum Theory in terms of explaining consciousness. First, we generally think of human qualities of consciousness as being different from some type of known energy. Second, Quantum Theory, in its present form, is distance- and timedependent. Human consciousness, on the other hand, does not appear to be dependent on distance and time (Appendix 1). An expanded scientific reference frame is therefore needed that can begin to explain human consciousness and my experience with the Buddha relics.

As I reconsidered my experience of the Buddha relics, one aspect was an important clue. In a single afternoon, the space of our home appeared to change to a different or altered state. The best term that came to mind was that it was now sacred. Now, we do not normally consider living rooms holy! What changes in the nature of the space where and when the relics are displayed? Although this line of questioning may appear incongruous to the basic question of the nature of the relics, it is important to remember that all that is manifest is basically floating, existing in the space, or physical vacuum. It is empty space that allows us to discern any content at all, and it is the seemingly only air-filled empty space that is the most mysterious aspect of nature. It is only now being appreciated that the dominant energy in our Universe does not reside in normal matter or even dark matter or black holes. ${ }^{18}$ Rather, it is located in the physical vacuum ("empty space") located between the fundamental particles that make up the atoms and molecules ${ }^{18}$ (Appendix 2 ). Returning to the question, what is the nature of change of the physical space?

\section{Four Target Experiments that Spin Science on Its Head}

To try to answer the question we have presented above, we need to first review important experimental data that show human consciousness has measureable quantitative effects on physical matter. Tiller and colleagues set out to investigate the unstated assumption of science that no qualities of human consciousness, intention, emotion, mind, or spirit can significantly influence a well-designed experiment in physical reality. ${ }^{19}$ For each target experiment, Tiller chose a very novel two-stage procedure. In the first stage, specific human intention from a deep meditative state was first imprinted mentally and emotionally into a simple "host" electrical device. In the process of imprinting the device, Tiller and his team always considered the imprinting as a sacred process. In this way, their imprint statement could be considered as something like a prayer. Following is an example of the intention statement that was utilized in their water $\mathrm{pH}$ studies:

To activate the indwelling consciousness of this experimental space in order to condition it to a significantly higher electromagnetic gauge symmetry state. The special tuning for this state is to be such that the intention host device increases (decreases) the $\mathrm{pH}$ of the experimental water, in equilibrium with air, by one full $\mathrm{pH}$ unit compared to the control; i.e., to decrease (increase) the hydrogen ion, $\mathrm{H}+$ thermodynamic activity of this water by a factor of ten. ${ }^{19}$

In the second stage, this Intention Host Device (IHD) was utilized to produce effects in an in vivo or in vitro biological experiment merely by electrically turning the IHD "on" in the immediate vicinity of a continuously running target experiment. The target experiments were (1) to increase or (2) to decrease the $\mathrm{pH}$ of a standard water sample by one full $\mathrm{pH}$-unit without adding any chemical components (the measurement accuracy was $\pm 0.01 \mathrm{pH}$ units); (3) to significantly increase $(\sim 30 \%$ at $p<0.001)$ the in vitro chemical activity of the liver enzyme alkaline phosphatase; and (4) to significantly increase the in vivo ratio of adenosine-5'triphosphate to adenosine diphosphate in the cells of fruit fly Drosophila melanogaster larvae so that they would have a significantly reduced $(\sim 25 \%$ at $p<0.001)$ larval developmental time to the adult fly stage. Astonishingly, all of these experiments were successful. ${ }^{19}$ In today's scientific worldview and understanding, there is a very low probability for any form of consciousness to have an effect on a physical experiment; furthermore, to actually imbed an intention in a host device, which then can be transported to another laboratory site and generate such effects, would be considered impossible. These results are nothing short of dramatic, but they are not overdramatic. The water +1 - $\mathrm{pH}$ target experiments have been successfully replicated by other independent investigators in their own laboratories. ${ }^{20}$ In many cases, the excess thermodynamic free energy of the experimental space was measured to more than double from its normally expected value of room temperature due to this type of space conditioning to a higher electromagnetic gauge symmetry state. ${ }^{19,21}$

There have been many published reports on the effects of intention in the parapsychology literature. However, Tiller's target experiments are unique in several important methodological ways: Imprinting a device allows an intention experiment to be transported from one laboratory to the next; there is an intention statement with the specification that the physical space is activated to condition it; the space where the IHD is kept is continuously monitored and recorded. However, the data of the target experiments, apart from replication by one independent group, remains primarily a single laboratory experience.

\section{Toward an Understanding of Symmetry of the Physical Space}

The above intention experimental data can appear lawful and understandable via a new framework; one that, crucially, includes an understanding of unique levels of symmetry of the physical space. During the replication process of their experimental data, Tiller and colleagues discovered a remarkable phenomenon. By continuing to use an IHD in the laboratory space over 3-4 months, the space itself became changed or "conditioned" and the state of that conditioning determined the robustness of the target experiments. ${ }^{19}$ For example, in the water $\mathrm{pH}$ experiments, after 2-3 months, oscillations in the laboratory air temperature, $\mathrm{pH}$, and direct current (DC) magnetic field polarity effects were observed. ${ }^{19}$ The experimental space changed from normal conditioning, 
also termed the U(1) EM (electromagnetic) gauge symmetry state to the next higher, SU(2) EM gauge symmetry state. ${ }^{22,23}$ From physics theory, the higher SU(2) EM gauge symmetry state is also a higher thermodynamic free energy per unit volume state. ${ }^{19}$ One signature of $\mathrm{SU}(2) \mathrm{EM}$ state is that magnetic monopoles appear to be accessed. This means that the $\mathrm{pH}$ of water is found to be different in the presence of a DC magnetic field with the North Pole facing the water versus the South Pole facing the water. Such anomalous results would never happen in U(1) gauge symmetry state space wherein only magnetic dipoles are accessed.

It was also found that the South Pole strengthens, whereas the North Pole weakens, various human muscle groups. Since muscle proprioceptors are intimately connected to the acupuncture meridians, this is evidence that the human body has already within it a higher symmetry state system, which is the acupuncture-meridian/chakra system. ${ }^{19}$ Table 1 summarizes some general characteristics of the $\mathrm{U}(1)$ and $\mathrm{SU}(2)$ gauge states. One consequence of $\mathrm{SU}(2)$ gauge state space is that there is an increased nonlocal interconnectivity between parts of a system, which has been termed macroscopic information entanglement. ${ }^{19,24}$

\section{Human Consciousness, Information Theory, and Thermodynamics}

We have just seen how human consciousness, in the form of intention, can raise the symmetry level of space to a higher or conditioned state, and in that conditioned space affect physical properties of matter. We can now consider the nature of consciousness. This brings up many philosophical points about what consciousness is. But if we go further and ask what consciousness does, we see that it manipulates information in the form of symbols such as language, numbers and mathematics, creativity such as art, and so on. Information theory was one of the most important scientific advances of the last half century, and it revolutionized the Internet age. ${ }^{25}$ We can talk to a friend on the telephone, view television pictures, and download photos of loved ones on the computer because of information theory. ${ }^{26}$ However, what is not well appreciated is that information is related to thermodynamics. Everything we encounter in our world, all processes are ultimately driven by thermodynamic free energy potentials (Appendix 3). Information, like matter and energy, is a basic principle of nature, and information through its relationship with thermodynamics can drive processes that we see in nature! Physicists are now beginning to recognize that information is more important than particles, strings, energy, and so on. ${ }^{27}$ Information gives rise to everything we see around us. Human intention and consciousness not only manipulate information but also are a source of information. Crucially, information is the bridge between energy and consciousness. We can expand the wellaccepted relationship of matter and energy that Einstein derived to include information as follows:

$$
\text { Mass } \leftrightarrow \text { Energy } \leftrightarrow \text { Information } \leftrightarrow \text { Consciousness }^{19}
$$

\section{A Working Hypothesis of the Buddha Relics}

The Buddhist Masters, through their spiritual qualities of unconditional love, and through intention (information) are able to imprint objects such as relics. Relics literally house and can transport the information of loving kindness; it is proposed that this powerful information can raise the gauge symmetry of space and affect the physical vacuum. It appears that not only is thermodynamic potential of a higher

Table 1. General Characteristics of the Normal and Second Higher-Gauge Symmetry States of the Physical Space ${ }^{a}$

\begin{tabular}{|c|c|c|}
\hline & $U(1)$ Gauge & SU(2) Gauge \\
\hline $\begin{array}{l}\text { Predominant medical scientific } \\
\text { model }\end{array}$ & Conventional medicine & Complementary and alternative medicine \\
\hline \multirow{2}{*}{$\begin{array}{l}\text { Fundamental constituent } \\
\text { substances }\end{array}$} & Electric atom-molecule & Magnetic wave information \\
\hline & Electromagnetic (EM) waves & $\begin{array}{l}\text { Magneto-electric (vacuum level analogue } \\
\text { of EM waves) }\end{array}$ \\
\hline Coordinates & Distance and time dependent & Reciprocal domain with frequency coordinates \\
\hline Mathematical properties ${ }^{\mathrm{b}}$ & Scalar and vector quantities & Vector and tensor quantities \\
\hline Signature of the space & Electric monopoles accessed & Magnetic monopoles accessed \\
\hline $\begin{array}{l}\text { Connectivity or entanglement } \\
\text { between parts of a system }\end{array}$ & Entanglement very locally present & $\begin{array}{l}\text { Nonlocal entanglement between parts of a } \\
\text { system can be present over huge distances }\end{array}$ \\
\hline $\begin{array}{l}\text { Information as source of } \\
\text { thermodynamic free energy } \\
\text { potential }^{\mathrm{C}}\end{array}$ & $\begin{array}{l}\text { Information has very small effects } \\
\text { generated using the Boltzmann's } \\
\text { constant; thermodynamic free } \\
\text { energy potential related to Gibbs } \\
\text { energy (see Appendix 3) }\end{array}$ & $\begin{array}{l}\text { Magnitude of the analogue Boltzmann's } \\
\text { constant theoretically of a higher order } \\
\text { and information effect sizes increase } \\
\text { very significantly; thermodynamic energy } \\
\text { potential has a contribution from change } \\
\text { in information and hence change in entropy } \\
\text { (see Appendix 3) }\end{array}$ \\
\hline
\end{tabular}

\footnotetext{
${ }^{a}$ Any physical measurement quantitatively consists of two parts, one from $\mathrm{U}(1)$ gauge state and one from $\mathrm{SU}(2)$ gauge state when there is space conditioning. Space conditioning may be present from imprinting with an intention host device or human biofields.

${ }^{\mathrm{b}}$ Scalar quantities can be described by one number such as temperature and $\mathrm{pH}$ values. Vector quantities have magnitude, direction, and phase angle to define the property at any one point in space, and tensor quantities have geometric mapping associated with any measurement at any one point in space.

'Boltzmann's constant is: $1.380650 \times 10^{-23}$ joules per Kelvin $\left(\mathrm{J} \mathrm{K}^{-1}\right)$.
} 
internal symmetry of space available wherever the relics are, but also that the atom-molecules within the space are ordered more coherently. Equipment such as a camera can become sensitized ${ }^{28,29}$ and capture such images as seen in Figure 1. The physical body's acupuncture-meridian system is aligned powerfully in the higher symmetry state, which, in turn, influences the chemistry and cellular function to a restorative milieu. Essentially, the relics appear to set up a higher symmetry state, so a whole new reality opens up and becomes one in which the mind and emotion domains can perform physical and higher dimensional work. In understanding the importance of space, physics begins to explain the seeming miraculous. There is harmony between science and spirituality.

A potential study to test the above hypothesis might involve imprinting electronic devices with the Buddha relic energy and studying the imprinted devices. Alternatively, the space in which a relic tour takes place could be monitored by measuring the excess thermodynamic free energy of the space wherein they are housed. These two proposed experiments are a reasonable beginning strategy to understand the sacred phenomena because they would not interfere with the relics themselves. Experiments to study gauge symmetries are difficult to conduct, and it may not be possible to prove that a $\mathrm{SU}(2)$ state is reached; however, it at least appears from the Tiller target experiments that the space has to be lifted above normal reality. It is possible that the Buddha relics set up even higher symmetry states than the SU(2) gauge state. The application of gauge theory to space conditioning is speculative at this stage. It is a work in progress, and much still needs to be discovered about the nature of space.

\section{Proposal of a New Paradigm for Medicine}

As a physician, I put forward some important theoretical implications of the science discussed above for the practice of medicine. In today's world, we generally function in the normal U(1) symmetry state: Here, randomized control trials and conventional therapeutics have a chance of working. The measuring instruments are also reliable. In the SU(2) gauge symmetry state, where much of complementary medicine is practiced, double-blind trials have little chance of success because of non-spacetime connectivity between all parts of the system space, including the doctor, patient, and treatment, and therefore an increase in what appears as the placebo effect. ${ }^{30,31}$ There are no fully satisfactory measurement systems for the $\mathrm{SU}(2)$ gauge symmetry state today; hence, clinical trials that are designed to assess efficacy of complementary medicine therapies are difficult to conduct.

We can also consider some practical clinical applications: Can we raise the symmetry of our medical clinics to a higher state, and maintain that state? We generally practice medicine using the "chemical medicine" system, whereby analysis of the chemistry state of the physical body is important and pharmaceuticals form our main therapeutics. Is it possible that in a higher symmetry state space our pharmaceuticals can begin to demonstrate different, more powerful, and less harmful potentials? The illustration in Figure 2 shows the conceptual scheme of such an environment. Using the above scientific thought, we can begin to seriously consider energy medicine and information medicine, which can powerfully

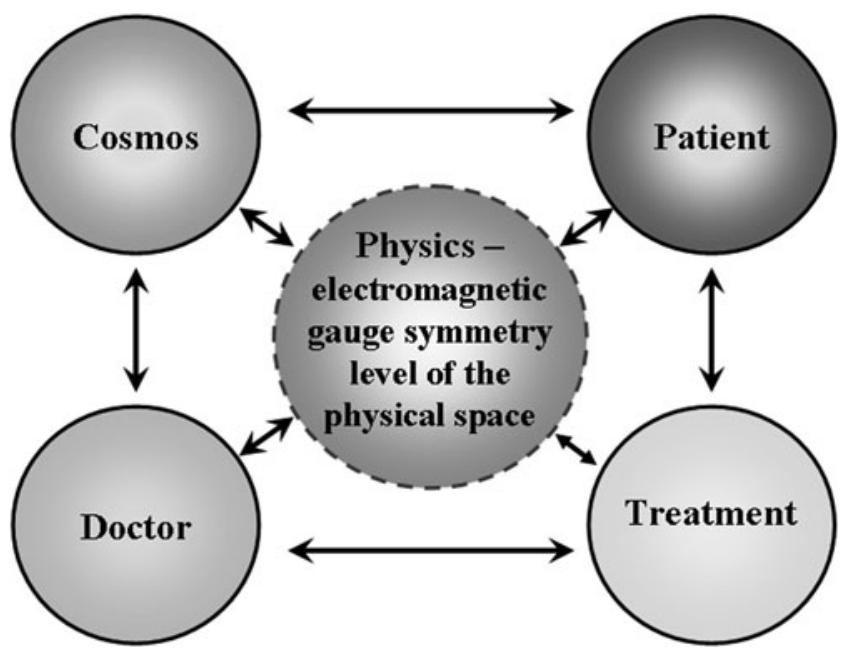

FIG. 2. A conceptual scheme for information medicine. ${ }^{19}$ There can be, and is, a profound connectivity between one part of nature and another and humans can potentially influence biological life. This is a simple diagram of the key factors involved in the general relationship. By intention, or information, raising the symmetry level of the clinic and hospital space can potentially positively affect all parts of the medical system: the doctor, patient, and treatment.

help conventional care. These potential applications are not in the distant future. ${ }^{32,33}$

\section{What Are the Buddha Relics Telling Us?}

The Buddha relics are a catalyst for our awakening. The relics and the science of physics show us that, as we become inner-self managed to a high degree, and focus our intentions, remarkable changes are possible in nature and also in ourselves. We are potential masters, not yet adept at this stage in our evolution. Through the practice of meditation and contemplation, we reduce the inner noise in ourselves and increase our inherent capacities to positively affect our reality. This is only the beginning of a discussion of science and spiritual realities and not the end. The identification of higher order physical laws does not remove, or "explain away" the effects of the Buddha relics; it does, however, provide an opportunity for science to investigate mechanisms of spiritual reality embodied in the human experience, and bring it to a pragmatic and practical use.

For me, an important lesson from the Buddha relics is to bring balance into my life, combining active service as a physician with regular periods of reflection. It is through my work as a physician that I express myself, and my love for humanity. I must also take essential steps in my inner-self management or self-education. I leave this short paragraph by a wonderful spiritual teacher, Vimala Thakar, on the need for self-education:

Spiritual life is a question of total revolution....Are we willing to let the change occur? In daily life the deeply rooted habit structure begins to move-and before you become aware of it-it starts to operate. For thousands of years the human race has been living that way. This is an effort to step out of that cycle. But the physical system requires time to get tuned in, or to stand the intensity and depth of that total awareness. It might slip back into the old habit structure if 
one has not re-educated the physical and mental structure, and there has not been an inner order; orderliness purifies. So, if that has not been done, if the physical has not been made sensitive and alert, then there is a chance that it slips back. But supposing it has been taken care of and yet one slips back into the old habits - it doesn't matter-if one is aware that one is slipping back, it is one step ahead. By such an inner voyage, one is carrying the whole human race within oneself. As there is the past contained in us, the total human past, one step that the individual takes in this direction of a new dimension of consciousness, a new dimension of life, means the whole humanity being carried in that direction. ${ }^{34}$

\section{Acknowledgments}

I am deeply grateful to Dr. William A. Tiller, Professor Emeritus of Stanford University Department of Materials Science and Engineering, for helping me with my physics, for spending many an hour discussing the Buddha relics over cups of tea, and for his courage in undertaking human intention research. I would also like to thank the Maitreya Project organizers for the remarkable opportunity they are bringing to people throughout the world; and for giving me and my family a rare opportunity to host the holy relics and to assist so many wonderful people in the short span of 3 days. Finally, I would like to express my thanks to my patients, who encouraged me to write of my spiritual experiences and scientific adventures in physics.

\section{Disclosure Statement}

No competing financial interests exist.

\section{References}

1. Barnes PM, Powell-Griner E, McFann K, Nahin RL. Complementary and alternative medicine use among adults: United States, 2002. Adv Data 2004;343:1-19.

2. Masters KS, Spielmans GI. Prayer and health: Review, metaanalysis, and research agenda. J Behav Med 2007;30:329-338.

3. Everly GS Jr, Benson H. Disorders of arousal and the relaxation response: Speculations on the nature and treatment of stress-related diseases. Int J Psychosom 1989;36:15-21.

4. Chang BH, Casey A, Dusek JA, Benson H. Relaxation response and spirituality: Pathways to improve psychological outcomes in cardiac rehabilitation. J Psychosom Res 2010; 69:93-100.

5. Kabat-Zinn J. An outpatient program in behavioral medicine for chronic pain patients based on the practice of mindfulness meditation: Theoretical considerations and preliminary results. Gen Hosp Psychiatry 1982;4:33-47.

6. Levin J. How faith heals: A theoretical model. Explore (NY) 2009;5:77-96.

7. Pace TW, Heim CM. A short review on the psychoneuroimmunology of posttraumatic stress disorder: From risk factors to medical comorbidities. Brain Behav Immun 2011; 25:6-13.

8. Kutz I, Borysenko JZ, Benson H. Meditation and psychotherapy: A rationale for the integration of dynamic psychotherapy, the relaxation response, and mindfulness meditation. Am J Psychiatry 1985;142:1-8.

9. Lazar SW, Bush G, Gollub RL, et al. Functional brain mapping of the relaxation response and meditation. Neuroreport 2000;11:1581-1585.
10. Davidson RJ, Lutz A. Buddha's brain: Neuroplasticity and meditation. IEEE Signal Process Mag 2008;25:176-174.

11. Thayer JF, Sternberg EM. Neural aspects of immunomodulation: Focus on the vagus nerve. Brain Behav Immun 2010;24: 1223-1228.

12. Pert CB, Dreher HE, Ruff MR. The psychosomatic network: Foundations of mind-body medicine. Altern Ther Health Med 1998;4:30-41.

13. Radin D, Hayssen G, Walsh J. Effects of intentionally enhanced chocolate on mood. Explore (NY) 2007;3:485-492.

14. Maitreya Project. 2011. Online document at: www.maitreya project.org/en/project/index.html Accessed August 10, 2011.

15. Lederman L. The God Particle. If the Universe Is the Answer, What Is the Question? Boston and New York: Mariner Book, Houghton Mifflin Company, 2006.

16. Stapp H. Mindful Universe. Berlin and Heidelberg: Springer-Verlag, 2007.

17. Miller AI. Erotica, Aesthetics and Schrödinger's wave equation. In: Farmelo G, ed. It Must Be Beautiful: Great Equations of Modern Science. London: Granta Publications, 2003:110-131.

18. NASA. Dark Energy, Dark Matter. 2011. Online document at: http://science.nasa.gov/astrophysics/focus-areas/what-isdark-energy/ Accessed March 30, 2011.

19. Tiller WA. Psychoenergetic Science: A Second Copernicanscale Revolution. Walnut Creek, CA: Pavior Publishing, 2007.

20. Pajunen GA, Purnell MJ, Dibble WE Jr, Tiller WA. Altering the acid/alkaline balance of water via the use of an intention-host device. J Altern Complement Med 2009; 15:963-968.

21. Tiller WA, Dibble WE. Toward general experimentation and discovery in conditioned laboratory and complementary and alternative medicine spaces: Part v. Data on 10 different sites using a robust new type of subtle energy detector. J Altern Complement Med 2007;13:133-149.

22. Moriyasu K. An Elementary Primer for Gauge Theory. Singapore: World Scientific Publishing Co., 1983.

23. William A. Tiller Foundation. White Paper XIX. Towards Understanding the Internal Symmetries of Nature: Gauge Symmetry States. April 29, 2011. Online document at: www.tiller.org Click on White Papers and choose by title. Accessed April 2011.

24. Tiller WA, Dibble WE, Orlando G, et al. Toward general experimentation and discovery in conditioned laboratory spaces: Part IV. Macroscopic information entanglement between sites approximately 6000 miles apart. J Altern Complement Med 2005;11:973-976.

25. Brillouin L. Science and Information Theory, 2nd ed. New York: Academic Press, 1962.

26. Aleksander I. Understanding information, bit by bit. Shannon's equations. In: Farmelo G, ed. It Must Be Beautiful. Great Equations of Modern Science. London and New York: Granta Books, 2003.

27. Vedral V. Decoding Reality. The Universe as Quantum Information. Oxford and New York: Oxford University Press, 2010.

28. Ledwith M, Heinemann K. The Orb Project. New York: Simon and Schuster, 2007.

29. Tiller WA. Science and Human Transformation: Subtle Energies, Intentionality and Consciousness. Walnut Creek, CA: Pavior Publishing, 1997.

30. Tiller WA. On understanding the very different science premises meaningful to CAM versus orthodox medicine: Part II. Applications of Part I fundamentals to five different 
space-time examples. J Altern Complement Med 2010;16: 507-516.

31. Tiller WA. On understanding the very different science premises meaningful to CAM versus orthodox medicine: Part I. The fundamentals. J Altern Complement Med 2010; 16:327-335.

32. Kohane MJ, Tiller WA. Biological processes, quantum mechanics and electromagnetic fields: The possibility of deviceencapsulated human intention in medical therapies. Med Hypotheses 2001;56:598-607.

33. Reed CR. The Effect of Intention on Decreasing Anxiety and Depression Utilizing Intention Imprinted Devices. Bolivar, MO: Holos University Graduate Seminary, 2005.
34. Thakar V. Contact with Vimala Thakar. On the need for self-education. In: Foundation VT, ed. Blaricum, Holland, 1977:3.

Address correspondence to: Nisha J. Manek, MD Division of Rheumatology Department of Medicine Mayo Clinic

200 First Street SW

Rochester, MN 55905

E-mail: njmanek@aol.com 


\section{Appendix 1. Differential Equations and the Mathematical Formulations to Describe Processes Occurring in Physical Nature}

Equations are classified in several different ways. One is by their degree. In linear equations, so-called because they describe straight lines (examples include $4 x+3 y=11$ and $y=3 x-8)$, the unknown numbers, represented by the letters $x$ or $y$, are not raised to any power and are said to be of the first degree. When the unknown is squared, the equation is called quadratic; when cubed, it is a cubic equation; after that it is an equation of the fourth, fifth, sixth degrees and so on.

When the solution to an equation is not a number but a function-when it is said to contain "derivatives"-it is called a differential equation. Differential equations arise specifically whenever a deterministic relation involving some continuously varying quantities and their rates of change in space and time (expressed as derivatives) are known or postulated. A deterministic system is a system in which no randomness is involved in the development of future states of the system. Differential equations form the mathematical foundation for classical and quantum physics. Some wellknown examples of second-order differential equations are Newton's second law of dynamics, Maxwell's equations in electromagnetism, and the Schrödinger wave equation. For normal things, second-order distance-time-dependent differential equations are adequate to explain behavior in nature. However, we cannot apply differential equations to consciousness. First, because we do not have an agreed-upon definition for consciousness; second, we have no way to quantify it; and third, we have, to date, shown no way to measure its distance- or time-dependence. Consciousness has not been specifically proven, and it is impossible to prove its nature using the current mathematical formalism of Quantum Theory (QT). This is an important distinction because it is generally believed that QT explains consciousness. To understand consciousness, if it was distance-time dependent, would lawfully require the specific use of a high-order distance-time-dependent differential equation with some force-field within it that is distance-time dependent, plus the appropriate number of specific boundary conditions. At this time, physics does not have a set of equations to utilize.

This leads into another issue that is often thought to represent human consciousness, and it is the nervous and neurohormonal systems of the body. The nervous system can be primarily considered only as "wiring" in the body. A nerve impulse travels along the length of such a wire, and this movement is distance-time dependent. But it does not follow a high order differential distance-time equation because that is what would be required to at least rationalize the quality we might call consciousness. It does not make that quality "consciousness." These distinctions are made here so that the reader can begin to appreciate the precision and limits of mathematics.

\section{Appendix 2. What Is the Physical Vacuum and How Does It Differ from Zero-Point Energy?}

In 1929, Paul Dirac offered a new model of electrons as coming from empty space. In essence, what Dirac proposed was a radically new conception of "empty" space which he said was, in reality, full with negative-energy qualities. Since the ordinary state of "empty" is far from empty, physicists now use the term "vacuum," which means the absence of physical substance. In Dirac's proposal, the vacuum is full of substance that can yield negative-energy electrons. This makes the vacuum a medium with dynamical properties of its own. For example, photons (light energy) can interact with the vacuum and, if photons with enough energy are provided, then this vacuum substance can absorb one of these photons, eject a negative electron, and leave a positive "hole." The physical vacuum is very important in understanding the laws of physics, and provides us with a frame in which the world is embedded. Quantum Theory has established this physical vacuum as a chaotic, virtual particle sea of boundless energy (energy density equivalent to approximately $10^{94} \mathrm{~g} / \mathrm{cm}^{3}$ ) at the quantum relativity level. A number of this magnitude is difficult for us to grasp, but if we use Einstein's equation: $\mathrm{E}=\mathrm{mc}^{2}$ then, in practical terms, the energy contained in the vacuum portion of a single hydrogen atom can be calculated and found to exceed, by a trillion times, all of the energy of the planets and stars combined in our cosmos! The physical vacuum state is difficult to detect under usual experimental conditions since it appears to function in a domain of reality with reference coordinates beyond space and time, but it is generally agreed that it is a source of almost infinite energy, as we have just seen.

The idea of Dirac's "hole" theory had its roots in solid-state physics. In a solid material, one has a reference or ground configuration of electrons, with the lowest possible energy at absolute zero temperature. Zero-point energy is a term that was first used to refer to the state of matter at absolute zero of temperature. Materials undergo fluctuations even at absolute zero temperature, which is a consequence of their wavelike or vibratory interactions. Zero-point energy is the vibrational state of electric atoms and molecules in physical material, which is electromagnetic (EM) in nature. We can detect it because our instruments are EM. All of quantum mechanics is EM. The physical vacuum is the absence of anything physical. It is not accurate to extrapolate zero-point energy, which is the ground EM state of materials, to the physical vacuum. If the physical vacuum is EM in nature, it would have been detected.

\section{Appendix 3. Information and the Second Law of Thermodynamics}

The second law of thermodynamics states that things in nature always flow from a higher free energy state to a lower free energy state. It is changes in Gibb's thermodynamic free energy that drives all the processes in nature that have so far been discovered. The Gibb's thermodynamic free energy function, $G$, is given by the following master equation:*

$$
G=P V+E-T\left(S_{0}-\sum_{j} \Delta I_{j}\right)
$$

where $P=$ pressure, $V=$ volume, $E=$ internal energy, $T=$ temperature, and $S=$ entropy, a measure of disorder, and $I=$ information. Thus, all the terms in changes of $G$, that is, $\Delta G$, $(P \Delta V, V \Delta P, \Delta E, T \Delta S$, and $S \Delta T)$ are equally important for producing significant changes in our world. Information is related to entropy; information change can be regarded as negative entropy change. The term in the brackets, $S_{0}$, is the

*William A. Tiller, PhD, Scottsdale, AZ [personal communication, February 2011 to June 2011]. 
original entropy, minus the sum, $\sum$, over all the different types (denoted: $j$ ), of information change (denoted: $\Delta \mathrm{I}_{j}$ ), active in the process. Consciousness manipulates information of all kinds: numbers, letters, pictures, symbols, etc., and a process producing an increase of information, $\Delta I$, leads to a decrease of thermodynamic entropy, $\Delta S$, by exactly the same amount where:

$$
\Delta S=-\Delta I \rightarrow \Delta G=-T \Delta S=T \Delta I
$$

Change in entropy, $\Delta S$, equals a negative change in information, $-\Delta I$, which yields a change in thermodynamic free energy, $\Delta G$. In turn, $\Delta G$ equals minus temperature, $T$, times the change in entropy, $\Delta S$, or temperature, $T$, times the change in information. 